\title{
Combined Treatment with Triptolide and Tyrosine Kinase Inhibitors Synergistically Enhances Apoptosis in Non-small Cell Lung Cancer H1975 Cells but Not H1299 Cells through EGFR/Akt Pathway
}

\author{
Xiaopei Tong, ${ }^{a, b}$ Pei Jiang, ${ }^{*, c}$ Yao Li, ${ }^{a, b}$ Lin Guo, ${ }^{a, b}$ Hui-min Zhang, ${ }^{a, b}$ Bi-kui Zhang, ${ }^{a, b}$ and \\ Miao $\operatorname{Yan}^{*, a, b}$ \\ ${ }^{a}$ Department of Pharmacy, the Second Xiangya Hospital, Central South University; No. 139 Middle Renmin Road, \\ Changsha Hunan 410011, China: ${ }^{b}$ Institute of Clinical Pharmacy, Central South University; No. 139 Middle Renmin \\ Road, Changsha Hunan 410011, China: and ${ }^{c}$ Institute of Clinical Pharmacy \& Pharmacology, Jining First People's \\ Hospital, Jining Medical University; No. 6 Jiankang Road, Jining 272000, China. \\ Received April 7, 2019; accepted May 21, 2019; advance publication released online May 29, 2019
}

Lung cancer is one of the most common malignant cancers in the world. Epidermal growth factor receptor tyrosine kinase inhibitors (EGFR-TKIs) is a second- or third-line therapy for mutated non-small cell lung cancer (NSCLC). It usually becomes drug resistance after a period of treatment. Triptolide (TPL) is an epoxy diterpenoid lactone compound extracted from Tripterygium wilfordii Ноок. F. and many studies demonstrated that TPL has a synergistic effect when combined with chemotherapy drugs. In this research, we plan to evaluate the combined effect of TPL and EGFR-TKIs (Gefitinib, Erlotinib, and Icotinib) and investigate the possible mechanisms. 3-(4,5-Dimethylthiazol-2-yl)-2,5-diphenyltetrazolium bromide (MTT) assay was conducted to detect the cell viabilities, combined effect was evaluated by Combination Index. Molecular docking study was used to predict the binding ability of TPL. The expression of proteins was detected by Western blot. MTT results showed TPL had synergistic effect with three EGFR-TKIs at different concentrations on H1975 cells but not on H1299 cells. Molecular docking study demonstrated that TPL with T790M/ L858R EGFR can form a more stable compound than that with wild type EGFR. Western blot results showed TPL inhibited the EGFR/Akt pathway and increased the expression of Bax and the ratio of Bax and Bcl-2 in H1975 cells. In conclusion, TPL had synergistic effect with three EGFR-TKIs on H1975 cells but not on H1299 cells, which may be due to the binding ability of TPL and different-type EGFR. The synergistic effect of TPL on H1975 cells may be partly related to the inhibition of the EGFR/Akt pathway.

Key words triptolide; tyrosine kinase inhibitor; combined use; synergistic effect; molecular docking; apoptosis

\section{Introduction}

Lung cancer is one of the most common malignant cancers in the world. ${ }^{1)}$ Non-small cell lung cancer (NSCLC) accounts for about $80 \%$ in all lung cancers, and about $75 \%$ of patients have been found in the middle or advanced stage. Epidermal growth factor receptor (EGFR) has tyrosine kinase activity which can promote cell division and proliferation, usually activated abnormally or overexpressed in NSCLC. The discovery of mutations in the tyrosine kinase domain of EGFR gene in NSCLC ${ }^{2)}$ leaded to the research of molecular-targeted therapy by EGFR-tyrosine kinase inhibitors (TKIs), such as Gefitinib (GE) and Erlotinib (EL).

GE and EL, a second- or third-line therapy for mutated nonsmall cell lung cancer, are targeting therapies for EGFR 858 site mutation and often used to ease the disease progression of patients with advanced cancer in clinical practice. Icotinib (IC), an EGFR-TKI drug discovered in China, targeting the same sites as GE and EL, has been gradually used widely for NSCLC. ${ }^{3)}$ However, drug resistance still occurs nine to ten months after treatment with EGFR-TKIs, and some of them developed to $\mathrm{T} 790 \mathrm{M} / \mathrm{L} 858 \mathrm{R}$ resistance mutation, resulting in the poor treatment outcomes. ${ }^{4)}$ How to increase the sensitivity of drug-resistant NSCLC to EGFR-TKI is a worthy study.

Triptolide (TPL) is an epoxy diterpenoid lactone compound with strong cytotoxic effect which is extracted from the flower, leaf, and fruit of Tripterygium wilfordii Hook. F. ${ }^{5}$
Tripterygium wilfordii is a traditional Chinese medicine, used for the treatment of rheumatoid arthritis, nephritis, lupus erythematosus, thrombocytopenic purpura and so on. ${ }^{6}$ Different from the total exacts of Tripterygium wilfordii, TPL is more usually used for anti-cancer research because of its strong cytotoxicity. A large number studies have reported that TPL has strong anti-tumor activity against a variety of cancers such as leukemia, breast cancer, pancreatic cancer, and lung cancer. $^{7-10)}$ Some studies have shown that TPL also have effect on improving the anti-tumor effect of chemotherapy drugs such as 5-fluorouracil, cisplatin or doxorubicin ${ }^{11-13)}$ and so on, reduced the dosage and side effects of chemotherapy drugs and improved the efficacy of drugs.

Zhang et al. ${ }^{14)}$ has researched the combined effects under different subsequences of TPL and Gefitinib on H1975. Based on this, we plan to investigate the combined effects of TPL and three kinds of EGFR-TKIs from another experimental viewpoint, observe their combined effects, analyze the combined effect, and explore the underlying mechanisms from EGFR/Akt signaling pathway.

\section{Experimental}

Chemicals and Reagents Triptolide (TPL, purity >98\%) was obtained from Huateng pharmaceuticals-company (Hunan, China). Gefitinib (GF, purity $>99 \%$ ), Erlotinib (EL, purity $>98 \%$ ) and Icotinib (IC, purity $>98 \%$ ) were obtained from 
<smiles>COc1cc2ncnc(Nc3ccc(F)c(Cl)c3)c2cc1OCCCN1CCOCC1</smiles>

A. Gefitinib (GF)<smiles>C#Cc1cccc(Nc2ncnc3cc4c(cc23)OCCOCCOCCO4)c1</smiles>

C. Icotinib (IC)

E

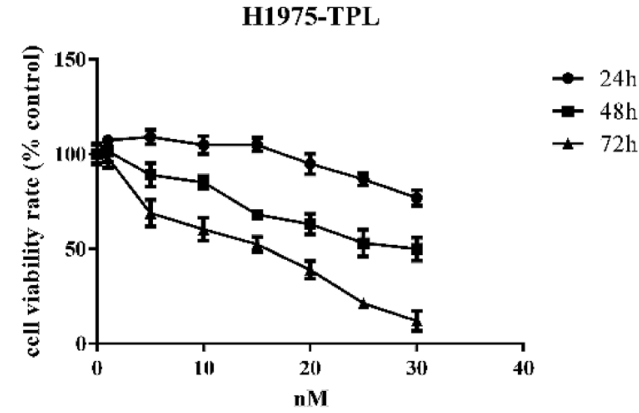<smiles>C#Cc1cccc(Nc2ncnc3cc(OCCOC)c(OCCOC)cc23)c1</smiles>

B. Erlotinib (EL)



D. Triptolide (TPL)

F

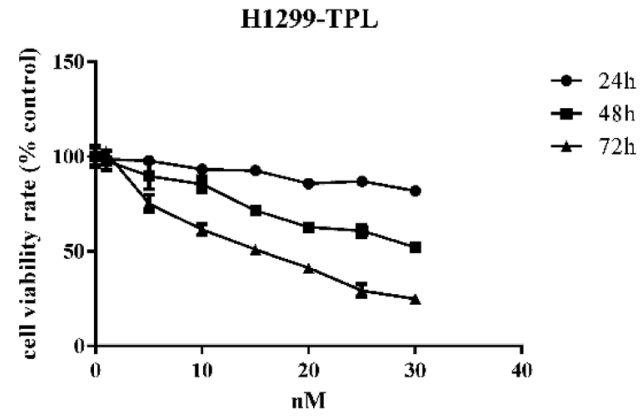

Fig. 1. The Structure of Gefitinib (A), Erlotinib (B), Icotinib (C) and Triptolide (D)

The cell viability rate (\% control) of H1975 cells (E) and H1299 cells (F) were measured by MTT assay when exposed to a series of increasing concentrations of TPL at 24,48 and $72 \mathrm{~h}$. Data are expressed as the mean \pm standard deviation (S.D.), $n=3$.

Bailingwei technology Co., Ltd. (Beijing, China). RPMI-1640 medium, phosphate-buffered saline (PBS) was obtained from Thermo Fisher Scientific, Inc. (Waltham, U.S.A.). Fetal bovine serum (FBS) was obtained from Biological Industries (Cromwell, U.S.A.). Dimethyl sulfoxide (DMSO) and 3-(4, 5-dimethylthiazol-2-yl)-2,5-diphenyltetrazolium bromide (MTT) was obtained from Sigma-Aldrich (St. Louis, MO, U.S.A.). Trypsin, penicillin, and streptomycin were obtained from Hyclone (Logan, U.S.A.).

Cell Lines and Cell Growth Human non-small cell lung cancer cell line H1975 and H1299 were obtained from CAS cell bank (Shanghai, China) and maintained in RPMI-1640 medium with $10 \% \mathrm{FBS}, 100 \mathrm{U} / \mathrm{mL}$ penicillin and $100 \mathrm{mg} / \mathrm{mL}$ streptomycin in the $37^{\circ} \mathrm{C}$ incubator with $5 \%$ carbon dioxide.

Cell Viability Effects of TPL, GF, EL, and IC on cell viability of H1975 and H1299 cells were determined by MTT assay. H1975 and H1299 cells were seeded at a density of $1 \times 10^{4}$ cells /well and plated onto a 96-well plate, with $200 \mu \mathrm{L}$ media per each well. Cells were exposed to different concentrations of TPL $(0,1,5,10,15,20,25,30 \mathrm{nM})$, GF $(0,1,2,4$, $8,12,16,20 \mu \mathrm{M}), \mathrm{EL}(0,1,2,4,8,12,16,20 \mu \mathrm{M})$ or IC $(0$, $1,2,4,8,12,16,20 \mu \mathrm{M})$ for 24,48 , and $72 \mathrm{~h}$. Subsequently, $20 \mu \mathrm{L} 5 \mathrm{mg} / \mathrm{mL}$ MTT solution with PBS was added to each well (so each well has a concentration of $0.5 \mathrm{mg} / \mathrm{mL}$ of MTT) and incubated for $4 \mathrm{~h}$ at $37^{\circ} \mathrm{C}$ with $5 \%$ carbon dioxide. Re- moved MTT and replaced with $200 \mu \mathrm{L}$ DMSO per each well, shaken for $10 \mathrm{~min}$ at room temperature. The optical density of each well was measured at $540 \mathrm{~nm}$ by using a Multiskan MS Plate Reader (LabSystems, Inc.; Thermo Fisher Scientific, Inc., U.S.A.).

Combined Effect Analysis To study the combined effect of the combination treatment of the formulation, Combination Index (CI) analysis was undertaken. ${ }^{15)}$ The results of cytotoxicity were evaluated via the CI. The CI of a combination of drugs less than 1 indicates the synergistic effect, CI equal to 1 means additive effect, and CI greater than 1 means an antagonistic effect. To evaluate the CI value, both individual drugs and drug combinations treatment in a range of concentrations were performed on the cell lines as described above. Briefly, for each level of $\mathrm{Fa}$ (the fraction of affected cells), the CI values were calculated by the following equation:

$$
\mathrm{CI}_{\mathrm{X}}=(\mathrm{D})_{\mathrm{A}} /\left(\mathrm{D}_{\mathrm{X}}\right)_{\mathrm{A}}+(\mathrm{D})_{\mathrm{B}} /\left(\mathrm{D}_{\mathrm{X}}\right)_{\mathrm{B}}
$$

$(D)_{A}$ and $(D)_{B}$ represent the $\mathrm{CI}_{\mathrm{X}}$ value of compound alone, respectively. $\left(D_{X}\right)_{A}$ and $\left(D_{X}\right)_{B}$ represent the concentration of compounds in the combination system at the $\mathrm{CI}_{\mathrm{X}}$ value. The $\mathrm{CI}_{\mathrm{X}}$ values curves were drawn according to $\mathrm{Fa}$. Fa values between 0.2 and 0.8 are considered validate.

Molecular Docking Study The crystal structure of T790M/L858R EGFR and wild type EGFR were loaded from 
H1975-GF

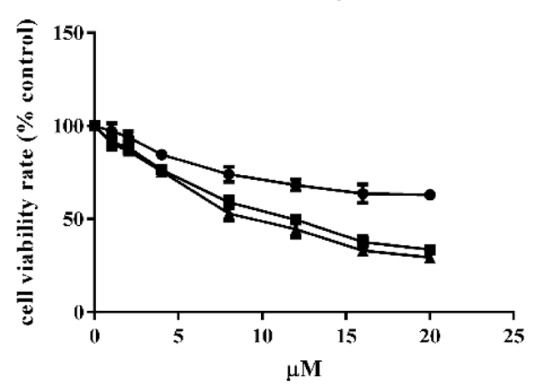

H1975-EL

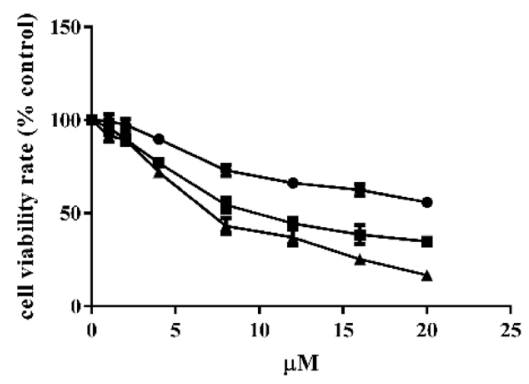

H1975-IC



H1299-GF

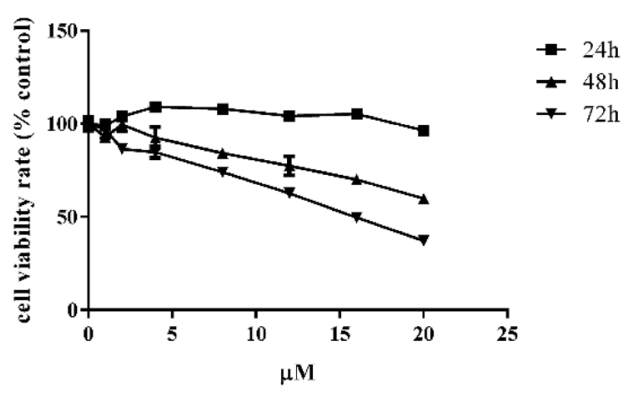

H1299-EL

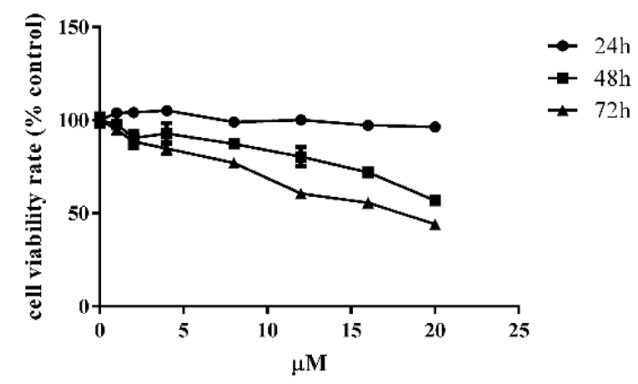

H1299-IC

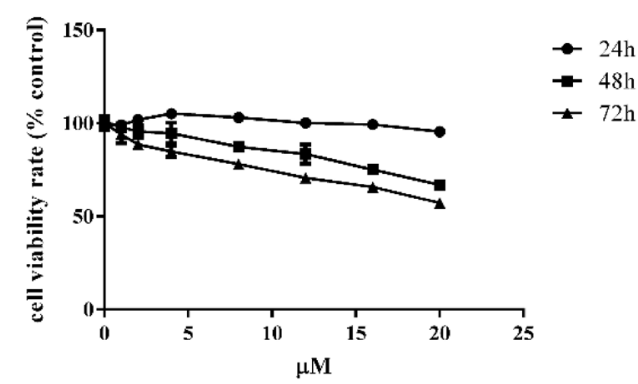

Fig. 2. The Cell Viability Rate (\% Control) of H1975 Cells (Left) and H1299 Cells (Right) Were Measured by MTT Assay When Exposed to a Series of Increasing Concentrations of GF, EL, and IC at 24,48 and $72 \mathrm{~h}$

Data are expressed as the mean \pm S.D., $n=3$.

the Protein Data Bank (PDB ID: 4LL0 and 4ZAU) in docking study. The molecular structure of TPL was optimized using MMFF94s force filed, and then T790M/L858R EGFR or wild type EGFR was protonated based on amber 99 force filed after removing water molecules. MOE-docking (MOE 2014.09 (Chemical Computing Group, Inc. Montreal, Canada)) was applied to identify the binding poses of TPL and EGFR. All docked poses of TPL were ranked based on the binding docking energies. The lowest energy conformation was chosen for binding modes analyses.

Western Blotting H1975 cells, treated with different concentrations of TPL in advance, were collected and prepared in cell lysis buffer for $30 \mathrm{~min}$ on ice and then centrifuged at $14000 \times \boldsymbol{g}$ for $30 \mathrm{~min}$ at $4^{\circ} \mathrm{C}$. The protein concentrations of the samples were determined by the Pierce BCA protein assay kit (Servicebio, G2026, Wuhan, China) according to the manufacturer's instructions. Protein was loaded and fractionated by $12 \%$ sodium dodecyl sulfate-polyacrylamide gel electrophoresis (SDS-PAGE) gel (Servicebio, G2003, Wuhan, China) at $80 \mathrm{~V}$ for $15 \mathrm{~min}$, and transferred to nitrocellulose membranes (Millipore, ISEQ00010, U.S.A.). Membranes were incubated with primary antibodies of EGFR (Abcam, ab52894), p-EGFR (Abcam, ab40815), Akt (Abcam, ab8805), p-Akt (Affinity,
AF0908), Bcl-2(Abcam, ab692), Bax (Abcam, ab32503), and $\beta$-actin (Servicebio, GB12001, Wuhan, China) overnight at $4^{\circ} \mathrm{C}$. Then incubated with the appropriate conjugated to a bovine-anti-mouse immunoglobulin and G-horseradish peroxidase-conjugated secondary antibodies (cat no., sc-2371; dilution, 1:2000; Santa Cruz Biotechnology). Membranes were detected using electrochemiluminescence detection system (Bio-Rad Laboratories, Inc., Hercules, CA, U.S.A.) and observed using a luminescent image analyzer (Image Quant LAS4000 mini, GE Healthcare, Sweden).

Statistical Analysis Data are presented as the mean \pm standard deviation and analyzed using GraphPad Prism 6. CDI value was calculated by Office Excel. Two-tailed Student's $t$-test was used for comparison between two groups, and $p<0.05$ was considered to indicate a statistically significant difference.

\section{Results}

TPL Showed Stronger Cellular Inhibition than GF, EL, and IC on H1975 and H1299 Cell Lines In the first step of the experiment, to determine the concentration of drugs in subsequent experiments, the inhibition effect of four drugs on H1975 and H1299 cells were detected. The structural formula of EGFR-TKIs and TPL were shown in below (Figs. 1A-D). 


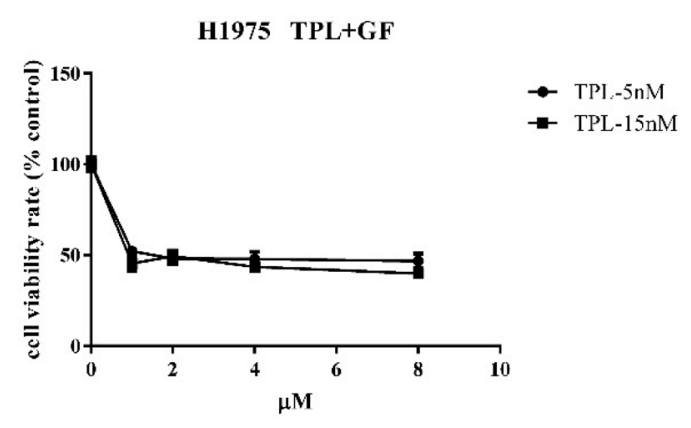

H1975 TPL+EL

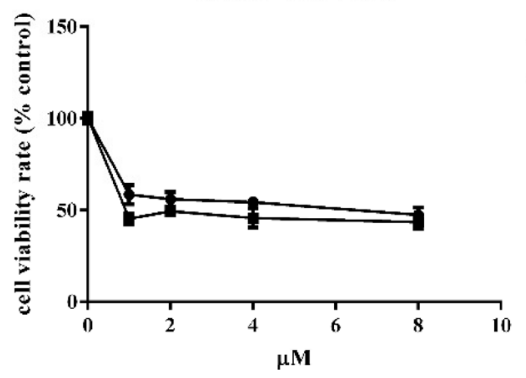

H1975 TPL +IC

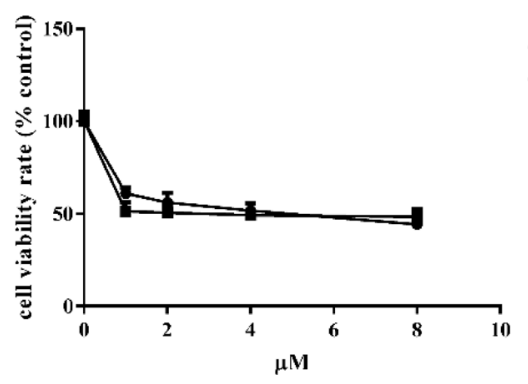

H1975 TPL+GF

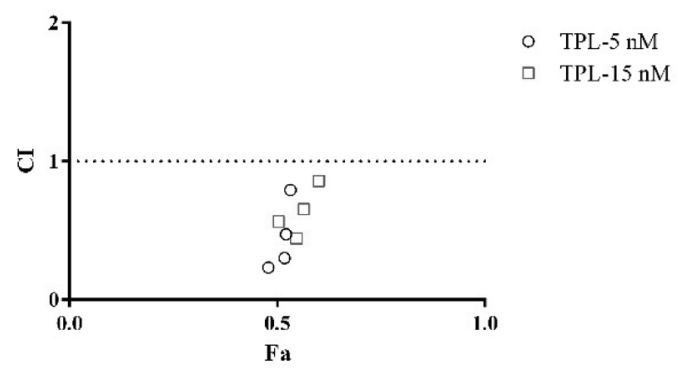

H1975 TPL+EL



H1975 TPL+IC

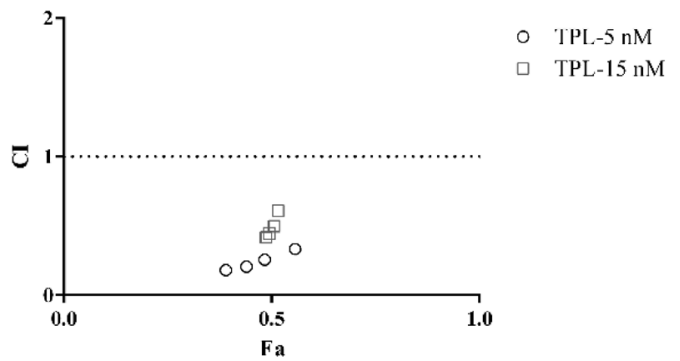

Fig. 3. Cell Viability Rate (\% Control) of H1975 Cells Were Measured by MTT Assay When under Combined of TPL and GF, or EL, or IC at $48 \mathrm{~h}$ (Left)

CI values were calculated and show on the matching right side. Data are expressed as the mean \pm S.D., $n=3$.

MTT results showed that TPL had the strongest inhibitory effect on H1975 and H1299 cells among four compounds, which can achieve a strong inhibitory effect of cell viability at a very small dose (Figs. 1E, $1 \mathrm{~F}$ and 2).

TPL Showed Synergistic Effect with GF, EL, and IC on H1975 Cell Line but Not on H1299 Cell Line Combined effects of TPL (5 or $15 \mathrm{nM}$ ) and GF or EL or IC $(0,1,2,4$, $8 \mu \mathrm{M})$ on H1975 cells were also determined by MTT assay. H1975 cells and H1299 cells were exposed to different concentration of TPL and GF or EL or IC for $48 \mathrm{~h}$. Then taken MTT assay. The results showed that at the concentration we detected, TPL had synergistic effects with GF or EL or IC (CI $<1)$ in H1975 cells, and most of their Fa values were clustered around 0.5 (Fig. 3). But for H1299 cells, there was no synergistic effects after the drug combination (Fig. 4). It should be noted that increasing the concentration of TPL or TKIs can only slightly increase the inhibitory effect on H1975 cells. The cell viability of H1975 cells and H1299 cells treated only with TPL ( 5 or $15 \mathrm{nM}$ ) showed in Table 1.

We also investigated the combined effect of lower drug concentrations. Gefitinib was selected as TKIs representative drug for the combined study on H1975 cells. Our results showed that when choosing lower concentrations of Triptolide $(1 \mathrm{nM})$ and Gefitinib $(0.5,1 \mathrm{nM})$, CI values did not get lower
(1.38537, 1.56795), showing an antagonistic effect, and the cell inhibition rates were too low which do not have practical significance (Table 2).

Drug-Resistant Mutant T790M/L858R EGFR Can Form a More Stable Complex with TPL than Wild Type EGFR The binding mode of TPL to T790M/L858R EGFR and wild type EGFR were shown in Fig. 5. The hydroxyl groups of TPL can form the hydrogen bond with the residue Thr854 of T790M/L858R EGFR in the complex. The interaction can provide the binding energy to stabilize the complex which means the TPL-T790M/L858R EGFR complex is a relatively stable composition. The docking score of them is -6.504 . While for wild type EGFR, there is no hydrogen bond between TPL and protein crystal structure, and the docking score is -4.46 , lower than TPL-T790M/L858R EGFR complex.

TPL Suppressed the EGFR/Akt Signaling Pathway and Induced Apoptosis H1975 cells were exposed to a series of continuous concentration of TPL $(0,5,15,20 \mathrm{nM})$ for $24 \mathrm{~h}$, then Western blot assays were taken. The results showed that TPL down-regulated the expression of EGFR, p-EGFR, Akt and p-Akt (Figs. 6 and 7), and upregulated the ratio of Bax and Bcl-2 especially at low concentrations (Fig. 8), which mean TPL have the potential capacity to induce cell apoptosis and suppress EGFR/Akt signaling pathway. 
H1299 TPL+GF

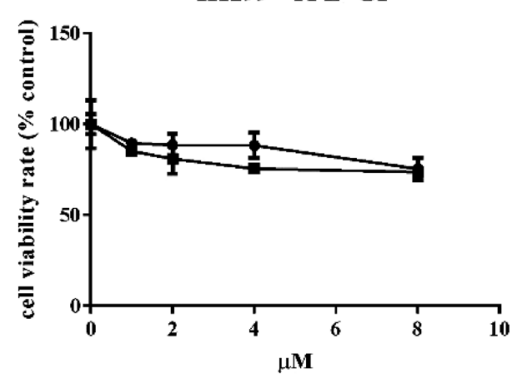

H1299 TPL+EL

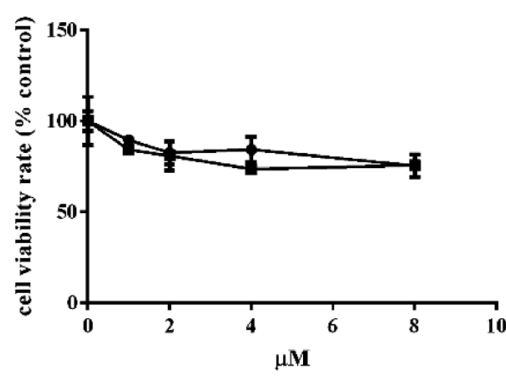

H1299 TPL+IC

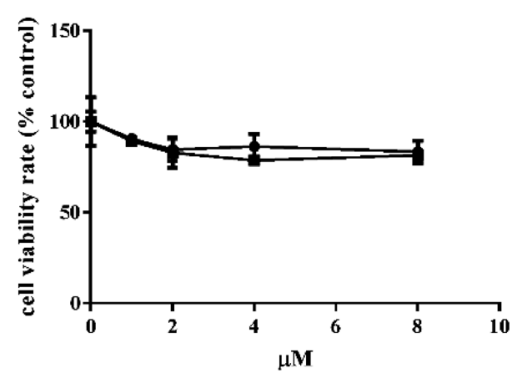

H1299 TPL+GF

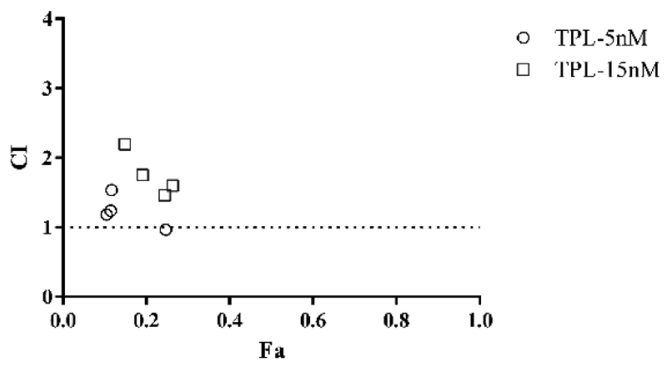

H1299 TPL+EL

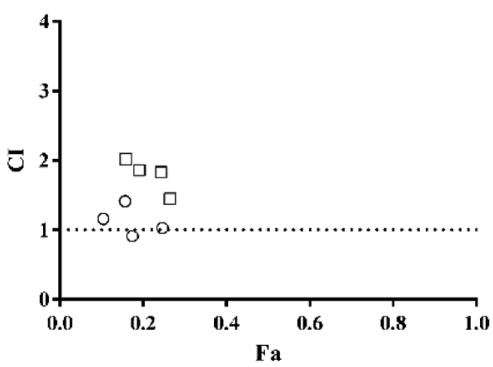

- TPL-5nM

TPL-15nM

TPL-5nM

TPL-15nM

TPL-5nM

TPL-15nM



Fig. 4. Cell Viability Rate (\% Control) of H1299 Cells Were Measured by MTT Assay When under Combined of TPL and GF, or EL, or IC at 48h (Left)

CI values were calculated and show on the matching right side. Data are expressed as the mean \pm S.D., $n=3$.

Table 1. Cell Viability (\% of Control) of H1975 Cells and H1299 Cells When the Concentratios of TPL are 5 and $15 \mathrm{nM}$

\begin{tabular}{lcc}
\hline \hline Cells & H1975 & H1299 \\
\hline Con. & & \\
TPL-5 nM & $89.00 \% \pm 6.1873$ & $89.73 \% \pm 7.1031$ \\
TPL-15nM & $68.24 \% \pm 2.351$ & $71.62 \% \pm 0.3724$ \\
\hline
\end{tabular}

Table 2. CI Values and Fa Values of TPL (1 nM) and GF at Lower Concentrations in H1975 Cells

\begin{tabular}{lcc}
\hline \hline Con. (TPL-1 nM) & GF- $0.5 \mu \mathrm{M}$ & GF-1 $\mu \mathrm{M}$ \\
\hline CI & 1.38537 & 1.56795 \\
Inhibition rate (\% of control) & $11.56 \%$ & $14.15 \%$ \\
\hline
\end{tabular}

\section{Discussion}

Tyrosine kinase inhibitors (TKI) is a kind of drugs that inhibit tyrosine kinase activity. GF, EL and IC are EGFR-TKIs for the treatment of NSCLC, which can compete with ATP and thus inhibit the activation of EGFR. TPL, because of its high content and strong physiological activity in Tripterygium wilfordii Hook. F, has been widely studied in anti-tumor, im- munomodulation, anti-inflammatory, ${ }^{16,17)}$ and other aspects. Interestingly, in the early literature, TPL was the target of attenuation and enhancement in the combined effects research. ${ }^{18)}$ In recent researches, TPL was used to combine with chemotherapeutic drugs to improve the therapeutic effect of chemotherapeutic drugs or increase the sensitivity of chemotherapeutic drug-resistant cells. As target-drugs almost act on a specific site, in this study, we shift the focus from chemotherapy drugs to target-drugs, to explore the synergies effect between TPL and EGFR-TKIs and the possible mechanisms.

We used the MTT assay and Combination Index (CI) analysis to evaluate the combined effects of EGFR-TKIs and TPL at different concentrations. The results showed that TPL had synergistic effect with GF, EL, and IC at different concentrations on H1975 but not H1299 cell line. Interestingly, increasing the concentration of TP or TKI did not have a great impact on the inhibitory effect of H1975 cells, but reduced their combined index. Since the effects by these concentrations of the compound in here seem to be saturated, we also investigated the combined effect at lower drug concentrations of TPL and GF on H1975 cells but the results showed an antagonistic effect, and the cell inhibition rates were too low which do not have practical significance. Therefore, according to our experimental results, relatively lower concentrations of TPL and 
A

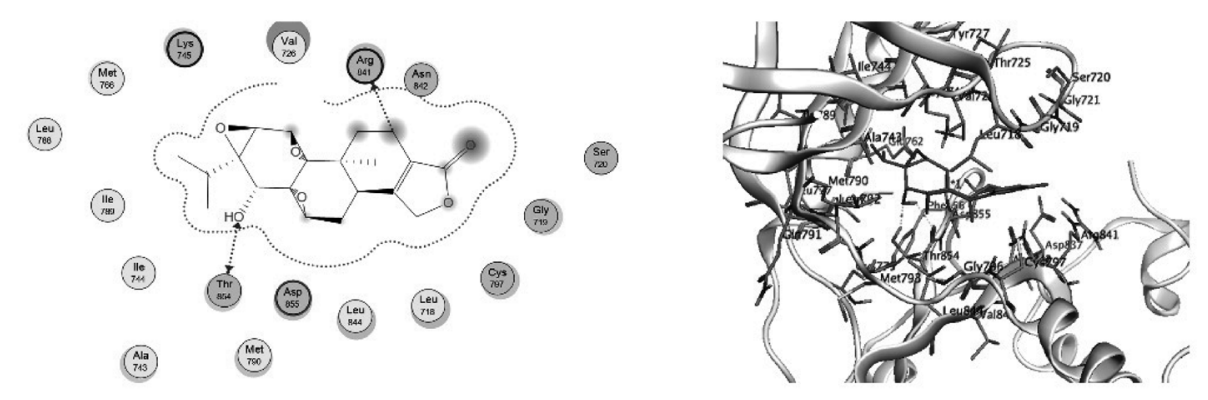

H1975 cells (T790M/L858R EGFR): -6.504

B

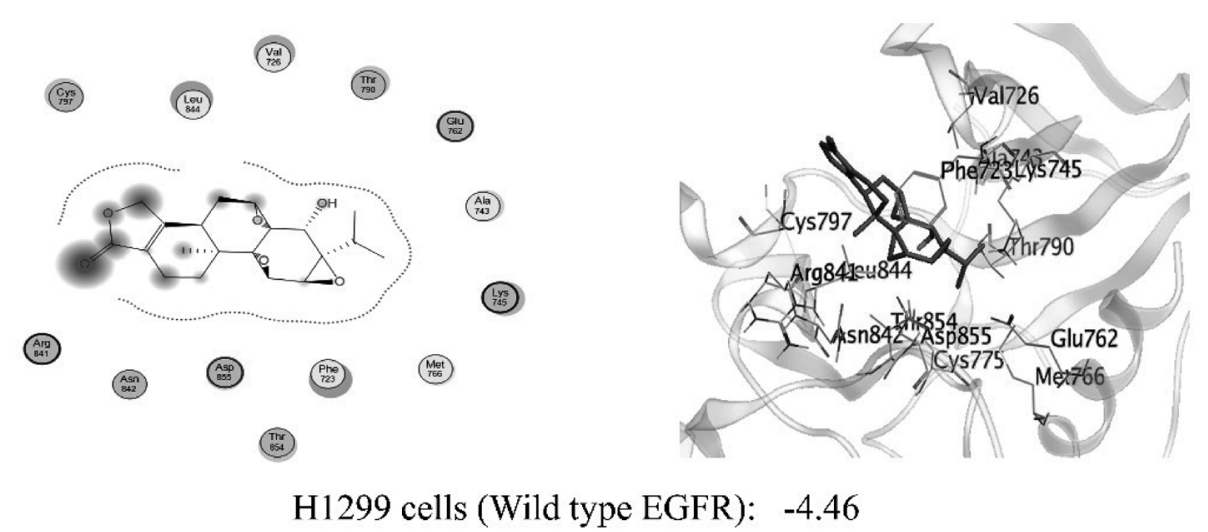

Fig. 5. Two-Dimensional and Three-Dimensional Representation of the Binding Mode of TPL with T790M/L858R EGFR (A) and Wild Type EGFR (B)

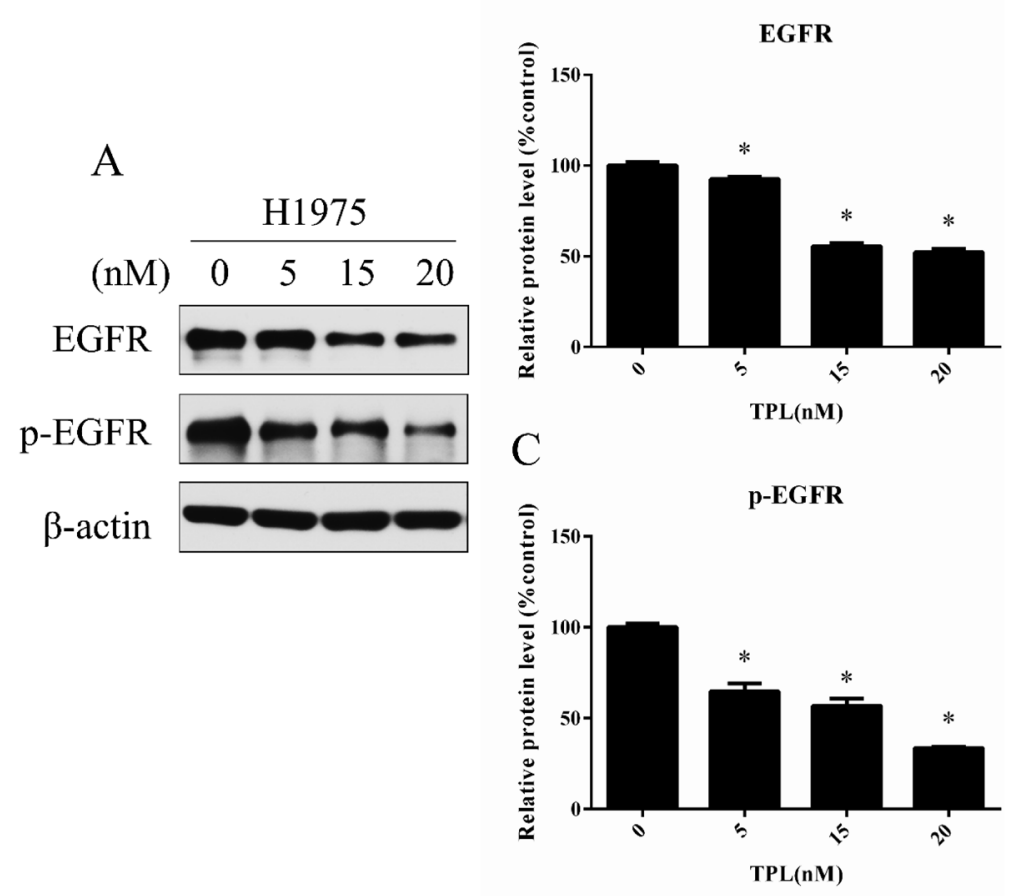

\section{B}

Fig. 6. (A) Representative Image of EGFR and p-EGFR Protein Determined by Western Blot

Cells were treated with TPL at indicated concentrations for $24 \mathrm{~h}$. Whole cell lysates were used for Western blot. Densitometric analysis of EGFR (B) and p-EGFR (C) by Western blot. Data are expressed as the mean \pm S.D., $n=3 . * p<0.05 v s$. NC group. 


\section{B}
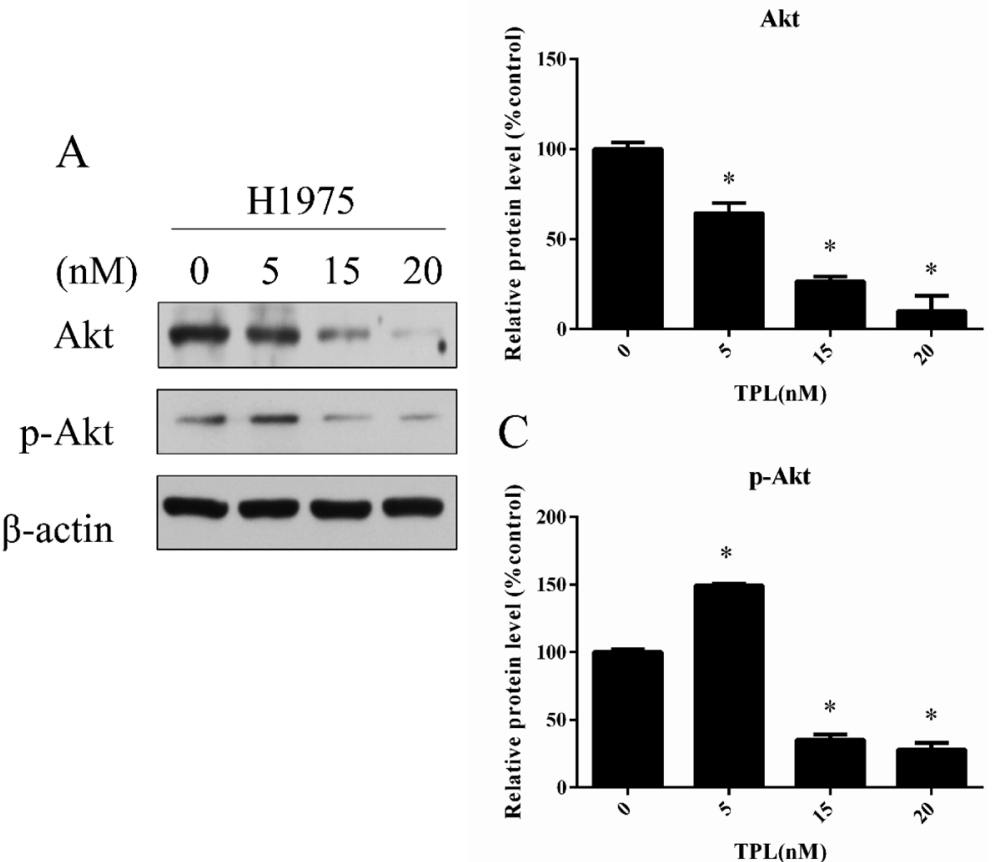

Fig. 7. (A) Representative Image of Akt and p-Akt Protein Determined by Western Blot

Cells were treated with TPL at indicated concentrations for $24 \mathrm{~h}$. Whole cell lysates were used for Western blot. Densitometric analysis of Akt (B) and p-Akt (C) by Western blot. Data are expressed as the mean \pm S.D., $n=3 .{ }^{*} p<0.05 v s$. NC group.

A

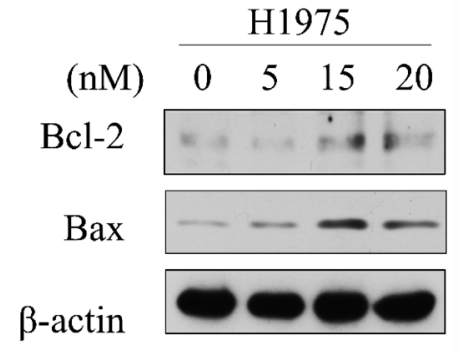

$\mathrm{D}$

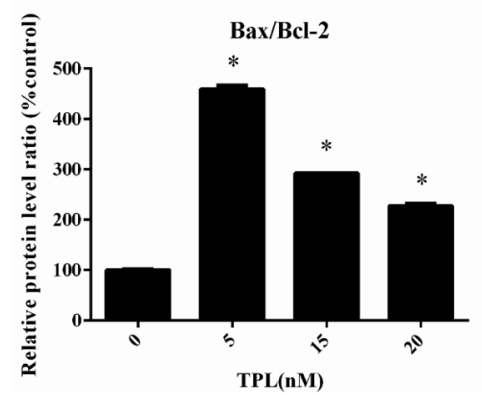

$\mathrm{B}$

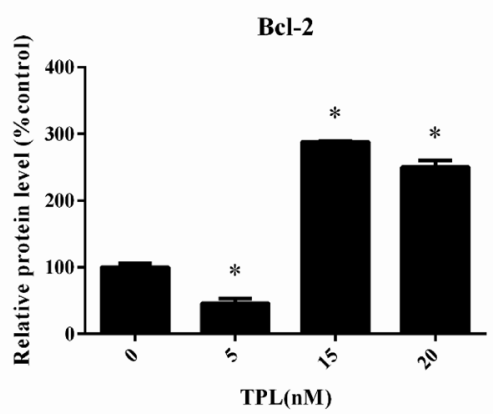

C

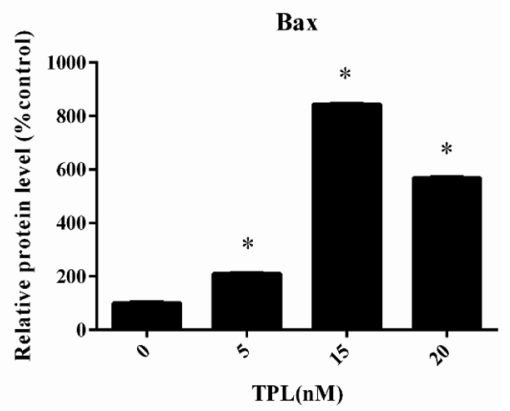

Fig. 8. (A) Representative Image of Bcl-2 and Bax Protein Determined by Western Blot

Cells were treated with TPL at indicated concentrations for $24 \mathrm{~h}$. Whole cell lysates were used for Western blot. Densitometric analysis of Bcl-2 (B) and Bax (C) and the ratio of Bax and Bcl-2 (D) by Western blot. Data are expressed as the mean \pm S.D., $n=3 .{ }^{*} p<0.05 v s$. NC group.

TKI is a better choice for combination therapy on H1975 cells.

We tested the affinity and the stability of the ligand of TPL to T790M/L858R EGFR and wild type EGFR by using computer simulated molecular docking technology. According to the molecular docking results in this study, we found the binding model of TPL-T790M/L858R EGFR is more stable than that of TPL-wild type EGFR. Combined with the data of the drug combination, it raises a possibility that there is a connection between the binding ability of TPL and EGFR and the combined effect. That is, the formation of a more stable 
complex of EGFR and TPL could contribute to the synergistic effect, otherwise, will be antagonism. While the possibility is still a conjecture triggered by the results of this experiment, it is indeed worth studying to find the relationship between synergism and EGFR phenotype, and more kinds of phenotypes of EGFR should be considered.

Another interesting finding is that Leuenroth and Crews ${ }^{19)}$ have used isotope tracer method to study the intracellular binding of TPL, and the results showed TPL is membrane localized, reversible and saturable. Although a more specific binding condition has not been mentioned in this literature, our experimental results could provide enlightenment that TPL may bind to the EGFR in cell membrane to exert its cytotoxic effect.

About the structural requirement of the compound when binding with EGFR, we could use for reference of the literature. Zhao et al. ${ }^{20}$ have screened more than two thousand kinds of plant-source compounds by molecular docking and found three compounds which have high combining ability with L858R EGFR, respectively is Matrine, Triptolide (docking score is -5.69), and Hydroxyjolkinolide B. There are many differences in the structure and combination way of those three compounds, ${ }^{20)}$ but we still can find some similar characteristics, which are also similar with TKIs. For example, they all have three to four six-member rings structure, the oxygen containing functional groups, and unsaturated bond. Therefore, we guess these characteristics may be related to their affinity for EGFR.

EGFR/Akt signaling pathway is a classic signaling pathway in NSCLC and can regulate cell growth activity. To investigate the mechanism of TPL on drug-resistant NSCLC cell lines, we examined EGFR and EGFR downstream pathway, which regulate the important activity like survival, proliferation, autophagy, and apoptosis in cells, to investigate the influence of TPL on it. The results of Western blot showed that TPL suppressed the EGFR/Akt signaling pathway and increased the expression of pro-apoptotic factor Bax and the ratio of Bax and Bcl-2. This suggests that TPL caused degradation of EGFR and its downstream proteins, and promotes cell apoptosis. As a compensative mechanism for EGFR-TKIs, which can partially explain its synergistic effects with GF, EL and IC.

\section{Conclusion}

From our experimental results, we found that TPL had synergistic effect with three EGFR-TKIs (GF, EL, and IC) on H1975 cells but not on H1299 cells, which may due to the binding ability of TPL and different-type EGFR. The synergistic effect of TPL on H1975 cells may be partly related to the inhibition of the EGFR/Akt pathway by TPL.
Acknowledgments This work was supported by the National Natural Science Foundation of China (No. 81573686), the Natural Science Foundation of Hunan Province, China (No. 2016JJ4098) and the Fundamental Research Funds for the Central Universities of Central South University (No. 2018zzts926).

Conflict of Interest The authors declare no conflict of interest.

\section{References}

1) Ferlay J., Colombet M., Soerjomataram I., Mathers C., Parkin D. M., Piñeros M., Znaor A., Bray F., Int. J. Cancer, 144, 1941-1953 (2019).

2) Smouse J. H., Cibas E. S., Jänne P. A., Joshi V. A., Zou K. H., Lindeman N. I., Cancer, 117, 67-72 (2009).

3) Shi Y., Sun Y., Ding C., Wang Z., Wang C., Wang Z., Bai C., Bai C., Feng J., Liu X., Li F., Yang Y., Shu Y., Wu M., He J., Zhang Y., Zhang S., Chen G., Luo H., Luo R., Zhou C., Zhou Y., Pang Q., Zhao H., Zhao Q., Gu A., Ling Y., Huang C., Han B., Jiao S., Jiao H., J. Thorac. Dis., 7, E468-E472 (2015).

4) Yamamoto H., Toyooka S., Mitsudomi T., Lung Cancer, 63, 315-321 (2009).

5) Kupchan S. M., Court W. A., Dailey R. G. Jr., Gilmore C. J., Bryan R. F., J. Am. Chem. Soc., 94, 7194-7195 (1972).

6) Luo D., Zuo Z., Zhao H., Tan Y., Xiao C., Fr. Medecine, 2019, in press.

7) Liu L., Li G., Li Q., Jin Z., Zhang L., Zhou J., Hu X., Zhou T., Chen J., Gao N., Cell Death Dis., 4, e941 (2013).

8) Li H., Pan G. F., Jiang Z. Z., Yang J., Sun L. X., Zhang L. Y., Acta Pharmacol. Sin., 36, 606-613 (2015).

9) Kim S. T., Kim S. Y., Lee J., Kim K., Park S. H., Park Y. S., Lim H. Y., Kang W. K., Park J. O., BMC Cancer, 18, 1103 (2018).

10) Reno T. A., Tong S. W., Wu J., Fidler J. M., Nelson R., Kim J. Y., Raz D. J., BMC Cancer, 16, 439 (2016).

11) Xu B., Guo X., Mathew S., Armesilla A. L., Cassidy J., Darling J. L., Wang W., Cancer Lett., 291, 200-208 (2010).

12) Wang X., Zhang J. J., Sun Y. M., Zhang J., Wang L. R., Li J. C., Liu H., Folia Biol, 61, 195-202 (2015).

13) Deng Y., Li F., He P., Yang Y., Yang J., Zhang Y., Liu J., Tong Y., Li Q., Mei X., Shu Z., Zhao Q., Mol. Carcinog., 57, 807-814 (2018).

14) Zhang X., Liu G., Liu H., Ma T., Zhongguo Fei Ai Za Zhi., 18, 599-609 (2015).

15) Chou T. C., Talalay P., Adv. Enzyme Regul., 22, 27-55 (1984).

16) Gao J. P., Sun S., Li W. W., Chen Y. P., Cai D. F., Neurosci. Bull., 24, 133-142 (2008).

17) Huang G., Yuan K., Zhu Q., Zhang S., Lu Q., Zhu M., Sheng H., Yu R., Luo G., Xu A., Mol. Immunol., 101, 210-220 (2018).

18) Wei Y. S., Ye Q. L., Jing J. J., He P., Long R. B., Cao Z. R., J Fujian Medical University, 2, 22-25 (1999).

19) Leuenroth S. J., Crews C. M., Chem. Biol., 12, 1259-1268 (2005).

20) Zhao G. F., Huang Z. A., Du X. K., Yang M. L., Huang D. D., Zhang S., Mol. Med. Rep., 14, 1132-1138 (2016). 